\title{
A new set of 272 pictures for psycholinguistic studies: Persian norms for name agreement, image agreement, conceptual familiarity, visual complexity, and age of acquisition
}

\author{
Leila Ghasisin • Fariba Yadegari • Mehdi Rahgozar • Ali Nazari • \\ Niloufar Rastegarianzade
}

Published online: 26 November 2014

(C) Psychonomic Society, Inc. 2014

\begin{abstract}
Pictures are often used in studies on memory, perception, and language; normative data are thus needed for such visual stimuli. In the present study, we aimed to obtain normative data for a set of 272 black-and-white pictures from middle-aged and elderly Persian speakers. A total of 206 volunteers were divided into two groups: a middle-aged (40-59 years old) group and an elderly (60 years old and over) group. The groups had similar characteristics in terms of education. Norms for every picture were developed to provide measures of name agreement, image agreement, conceptual familiarity, age of acquisition, and visual complexity. The results revealed that all of these measures vary with age, except for conceptual familiarity.
\end{abstract}

Keywords Persian language · Picture norms · Visual complexity $\cdot$ Age of acquisition $\cdot$ Familiarity $\cdot$ Image agreement $\cdot$ Name agreement

Electronic supplementary material The online version of this article (doi:10.3758/s13428-014-0537-0) contains supplementary material, which is available to authorized users.

\section{Ghasisin · F. Yadegari $(\bowtie)$}

Department of Speech Therapy, University of Social Welfare and

Rehabilitation Sciences, Tehran, Iran

e-mail: faribayadegari@yahoo.com

L. Ghasisin

Isfahan University of Medical Sciences, Isfahan, Iran

\section{Rahgozar}

Department of Statistics, University of Social Welfare and

Rehabilitation Sciences, Tehran, Iran

A. Nazari

Psychology Department, University of Tabriz, Tabriz, Iran

N. Rastegarianzade

Department of Speech Therapy, School of Rehabilitation, Shiraz

University of Medical Sciences, Shiraz, Iran
Naming is a key skill in interpersonal communication through language, which includes various cognitive processes, such as converting the visual stimuli into a conceptual representation, name retrieval, picture retrieval, and lexical production (Dell, Schwartz, Martin, Saffran, \& Gagnon, 1997). Anomia, which implies a naming impairment, is one of the most common deficits observed in different types of aphasia and other neurocognitive impairments. Picture-naming tests are frequently used in aphasiology studies and cognitive science research to examine naming ability (Tallberg, 2005), including event-related potential and functional magnetic resonance imaging studies (Severens, Van Lommel, Ratinckx, \& Hartsuiker, 2005). This task taps all of the stages of lexical production, including lexical access, phonological coding, and lexical information stored in the individual's memory in terms of both qualitative and quantitative aspects (Friedmann, Biran, \& Dotan, 2013).

A picture-naming test requires subjects to name the picture laid before them. Since pictures are different in terms of many details such as visual complexity and conceptual familiarity, normative data should be available for pictures to ensure accuracy of the test findings. In addition, it is highly important to control for the variables affecting naming. The relationship between a picture and its concept is not necessarily clear. A concept may be associated with several pictures. On the other hand, a picture may provoke several names. Until the end of the 1970s, no normative data were available on this issue, but then Snodgrass and Vanderwart (1980) presented a set of 260 standardized black-and-white pictures in English. These stimuli were selected on the basis of a number of variables that affect the memory process (Pompeia, Miranda, \& Bueno, 2001). Snodgrass and Vanderwart's method was enthusiastically welcomed not only in English, but also in other languages such as Greek (Dimitropoulou, Dunabeitia, Blitsas, \& Carreiras, 2009), Russian (Tsaparina, Bonin, \& Méot, 2011), Portuguese 
(Pompeia et al., 2001), Japanese (Nishimoto, Miyawaki, Ueda, Une, \& Takahashi, 2005), and Chinese (Weekes, Shu, Hao, Liu, \& Tan, 2007). Similar studies have also been conducted in Persian (Bakhtiar, Nilipour, \& Weekes, 2013) and Turkish (Raman, Raman, \& Mertan, 2014)

Normative data from picture-naming tasks usually appertain to name agreement, image agreement, conceptual familiarity, visual complexity, and age of acquisition. With the advent of speech production models, the impact of such factors on the speed and accuracy of naming can be justified (Levelt, Roelofs, \& Meyer, 1999). Word production in response to a visual stimulus up to the beginning of articulation includes at least the following processing stages: activation of structural knowledge stored in the subject regarding the object appearance, activation of semantic information, lexical retrieval, and phonological encoding. The above-mentioned measurements are capable of influencing these stages. Visual complexity and image agreement are interwoven with appearance features of the image, which is effective in recognition processes. Conceptual familiarity is effective in activating the concept and semantic representations. With respect to name agreement, frequency, and acquisition age, it can be argued that these measurements are effective at all lexical access stages (Laganaro, Di Pietro, \& Schnider, 2006).

Name agreement indicates the extent to which the subjects use the same word for a given picture (Dimitropoulou et al., 2009). This measurement is a strong predictor of naming difficulty and an important factor for studies on naming latency, name-picture matching, recalling, recognition, and phonological coding (Pompeia et al., 2001). Yet, image agreement clearly suggests the degree to which a given picture corresponds to the individual's mental representation. Studies have shown that pictures with high image agreement are named faster than those with low image agreement. According to Barry, Morrison, and Ellis (1997), the degree of image agreement could affect the identification of objects; thus, images that are closer to the mental picture are named faster. Still, it appears that image agreement exerts a more marked effect on naming black-and-white than on naming color pictures (Weekes et al., 2007).

Another important variable in the picture normalizing procedure is conceptual familiarity, which is referred to as the degree to which people are engaged in or think of a particular concept in their daily life (Tsaparina et al., 2011). Conceptual familiarity is an important predictor of naming reaction times, and it has been established that more familiar concepts are named faster (Hirsh \& Funnell, 1995). Pompeia et al. (2001) reported that the degree of familiarity exerts a direct effect on the activation of conceptual representations.

The effect of conceptual familiarity might be owing to the frequent contact with the object. The subject sees the object more frequently; hence, visual processes and access to semantic representation occur more quickly and easily. In other words, familiar objects are semantically richer than less familiar objects. This causes these objects to be more quickly and accurately recognized and more resistant to brain injuries (Cuetos, Aguado, Izura, \& Ellis, 2002).

Visual complexity can be defined as people's judgment of pictorial details. Some studies have shown that the more visually complex a picture is, the longer it will take to name it (Dimitropoulou et al., 2009). Visual complexity affects variables such as naming latency, tachistoscopic recognition threshold, and memorability (Pompeia et al., 2001). However, it seems that visual complexity is important in recognizing and naming colored pictures (Raman et al., 2014).

Age of acquisition is one of the factors that affect the speed of lexical retrieval and naming, and words learned at younger ages will be retrieved faster. Thus, there is a positive correlation between the age of acquisition and naming reaction times. Cortese and Khanna (2007) found that age of acquisition is the key factor in lexical naming and lexical decision performance. Moreover, age of acquisition is an important factor to predict the words that are retained and those that are lost in aphasic and semantic dementia patients. In such patients, it is likely that words learned first will be retained better (Cuetos, Samartino, \& Ellis, 2012). Morrison, Ellis, and Quinlan (1992) maintained that the age of acquisition affects the retrieval of the phonological word forms, for which they proposed two explanations. First, the age of acquisition affects the strength of relation between semantic and phonological representations. Second, the age of acquisition affects the intrinsic properties of phonological representations.

However, it seems that the age of an individual may affect the perception, judgment, and naming of images. Aging may cause changes in cognitive performance, including visuospatial and verbal working memory, long-term memory, and vocabulary (Park \& Gutchess, 2002). For example, Hodgson and Ellis (1998) indicated that elderly subjects committed more errors in naming and exhibited more semantic and visual errors than did young subjects. Furthermore, Sirois, Kremin, and Cohen (2006) contended that normative data should be provided for all age groups. However, the application of measurements whose normative data are essentially derived from young adults to those from different age groups may pose problems, especially when the subjects are old and suffer from cerebral lesion and neurodegenerative diseases such as Alzheimer's. Yoon et al. (2004) found a considerable difference in name agreement proportions between younger and older adults. Overall, although no norms are available with respect to middle-aged and elderly populations, such information is highly important because these people are more vulnerable to cognitive disorders and cerebral lesions. 


\section{The present normative study}

In the present study, we aimed to gather normative data pertaining to the ability of picture naming among middleaged and elderly Persian-speaking people. Bakhtiar et al. (2013) had already conducted a similar study on 18- to 29year-old people in Persian. Since middle-aged and elderly Persian-speaking people have not been studied in this regard, with the present study we aimed to provide an age-related normative data on the Persian picture-naming task.

Persian is the official language of Iran and is also spoken in other countries such as Afghanistan and Tajikistan. Currently, there are about 110 million Persian speakers in the world. Phonologically, Persian is made up of 29 segmental phonemes, including four pairs of stops, four pairs of fricatives, two nasals, one liquid, one trill, three glides, and six vowels. In Persian, a syllable does not begin with a vowel, and the syllable structure includes CV, CVC, and CVCC patterns. Persian also does not have initial clusters. In terms of morphology, Persian is an agglutinative language having many suffixes and few prefixes. Verbs reflect tenses and conditions and agree for person and number. Persian has no grammatical gender and no pronouns representing gender. The syntax of Persian is such that normal declarative sentences have the structure subject + object + verb. Thus, sentences can be a synthesis of optional subjects (pro-drop), appositives, and objects. Persian has the capability to create or make many words as derivatives (Windfuhr, 2009).

The present study was aimed at developing Persian norms for name agreement, image agreement, conceptual familiarity, visual complexity, and age of acquisition for middle-aged and elderly groups using a set of 272 black-and-white pictures.

\section{Method}

\section{Subjects}

A total of 206 volunteers were selected as subjects. The middle-aged subjects (including 51 women and 52 men) were $40-59$ years old $(N=103$, mean $=47.96, S D=5.554)$, and the elderly group (including 51 women and $52 \mathrm{men}$ ) were over 60 years old $(N=103$, mean $=67.66, S D=6.014)$. According to the Iranian system of education, the subjects were classified as illiterate, primarily literate, having diploma, or highly educated. All of the subjects were native Persian speakers and had a healthy cognitive status. Subjects with any medical, cognitive, or visual disorders were excluded from the study. Table 1 illustrates the socio-demographic characteristics of the subjects. Owing to the effect of education on the estimation of all measurements (Sirois et al., 2006), the two groups were compared in this regard, and no significant difference was observed $\left[\chi^{2}(1, N=206)=6.14, p=.02\right]$.

\section{Materials}

A set of 272 picturable concepts was selected on the basis of two criteria: lack of ambiguity and availability of their frequency information. Accordingly, concepts with frequencies available in a 1-million-word corpus (Hassani, 2005) were selected. Subsequently, pictures were provided for the selected concepts. A total of 79 pictures were selected from the Philadelphia naming tests, and 57 pictures were selected from the study by Cycowicz et al. (1997). The pictures were downloaded from the following websites: www.nyspi.cpmc. columbia.edu/nyspi/respaprs/picnorm.htm, and http://mrri. org/philadelphia-naming-test.

However, the remaining 136 pictures did not exist in the above-mentioned sources. The procedure for selecting the pictures was as follows: Three pictures were designed for each word by a graphic designer. Thus, each set of three pictures was specific to a single concept. The set of pictures was shown to ten normal subjects older than 40 years with sufficient levels of education and appropriate cognitive condition. The subjects were selected from the neighborhood and among relatives. To select the best picture, the criteria of Snodgrass and Vanderwart (1980) - that is, the degree to which the pictures were realistic, unambiguous, and sufficiently detailed as they would be in real worldwere employed for the subjects to judge. The subjects were asked to select the best picture in each set on the basis of the described criteria. The chosen picture was then given a score of 1 , and the unselected pictures received a score of 0 . Finally, the score of each picture was calculated and the pictures with the highest scores were selected.
Table 1 Socio-demographic characteristics of the subjects

\begin{tabular}{llllllll}
\hline \multirow{2}{*}{ Groups } & Gender & \multicolumn{6}{l}{ Education } \\
\cline { 2 - 5 } \cline { 5 - 7 } & Female & Male & & Illiterate & $\begin{array}{l}\text { Primarily } \\
\text { Literate }\end{array}$ & Diploma & $\begin{array}{l}\text { Highly } \\
\text { Educated }\end{array}$ \\
\hline Middle-aged & $51(49.5 \%)$ & $52(50.5 \%)$ & $5(4.9 \%)$ & $8(7.8 \%)$ & $53(51.4 \%)$ & $37(35.9 \%)$ \\
Elderly & $51(49.5 \%)$ & $52(50.5 \%)$ & $8(7.8 \%)$ & $12(11.7 \%)$ & $61(59.1 \%)$ & $22(21.4 \%)$ \\
\hline
\end{tabular}


The pictures were black and white with a size of 300 $\times 400$ pixels. A computer program was developed using the $\mathrm{C}++$ language to present the pictures to the subjects. The software could record the subjects' voices and store the data on every individual subject in a separate file. The software was developed with the aim of resuming the test from the point of stop if a subject's performance was interrupted. Every picture was displayed on the monitor for $30 \mathrm{~s}$ as the maximum time (Dell et al., 1997), and whenever the subject provided the answer, the picture was changed. The participation of elderly and illiterate people was the main reason for the 30-s picture appearance, because our aim was not to study reaction time, naming latency, or speed of naming, but just to find out whether a picture was or was not named correctly. So we did not put the elderly people under time pressure, and got them enough time to retrieve a name. The pictures were organized in such a manner that similar phonological and semantic categories did not occur together.

\section{Procedure}

All subjects completed the entire task. Since the whole experiment lasted for $5 \mathrm{~h}$ on average for each subject, the rating of each variable was conducted in a separate session. Thus, each subject completed five sessions of the experiment. In each testing session, the pictures were randomly presented to the subjects to be orally named. Each subject was tested individually. The subjects were instructed on how to respond to the stimuli. Since both illiterate and primarily literate individuals participated in the study, the responses were elicited orally. In all of the test stages, the examiner was present and the subjects' responses were written simultaneously with the voice recording. The examiner provided no feedback on the subjects' responses. The oral instructions were presented in accordance with the procedures established in a previous study (Bonin, Peereman, Malardier, Méot, \& Chalard, 2003).

The whole task involved four subtasks: name agreement, image agreement, conceptual familiarity, and age of acquisition. In the name agreement task, the subjects were instructed to utter the first name conjured up in their mind. The alternative names spoken by the subjects were recorded to be used in computing name agreement. If the subjects did not know the name of the picture, they responded with "I don't know." If they knew the picture's name but did not remember it (tip of the tongue), they responded with "I can't remember."

In the image agreement task, the subjects were asked to determine how similar their mental image was to the presented picture. In this regard, the examiner named the picture aloud for $5 \mathrm{~s}$ before it was visually presented to the subject. Within this 5-s interval, the subjects kept their eyes closed and tried to imagine the object. Then, they opened their eyes, looked at the presented picture on the screen, and decided the degree of agreement between these two pictures on a 5-point scale, ranging from 1 (least agreement) to 5 (strongest agreement).

In the conceptual familiarity task, the subjects were asked to determine the extent to which they were familiar or unfamiliar with the concept represented by the picture, in terms of a 5-point scale, with 1 denoting low familiarity and 5 indicating high familiarity. In the visual complexity task, the subjects evaluated the visual complexity of the pictures using a 5-point scale ranging from 1 (very simple) to 5 (very complex). The subjects were instructed to judge the visual complexity of the presented picture and not of the object in real life.

In the age-of-acquisition task, the subjects were asked to estimate the age at which they had learned the name of the picture. This estimation was based on a 5-point scale, as follows: $1=0-3$ years old, $2=4-6$ years old, $3=7-9$ years old, $4=10-12$ years old, and $5=$ over 12 years old. All the subjects completed the tasks in a friendly and comfortable environment. If the subjects decided to stop the task performance, the test was stopped until they consented to resume the tasks.

The following measurements were performed for each picture:

1. The modal name was determined - that is, the name that most of the subjects used to represent a given concept.

2. Name agreement was calculated - that is, the degree to which the subjects agreed on the name of a given picture. Two measurements were used for this purpose: namely, the proportion of people who used the same name, and $H$, an index that indicated the alternative names that the subjects used for a concept. Snodgrass and Vanderwart (1980) contended that the $H$ index (equation presented earlier) is a better measure to denote the diversity of the names used for a given concept, when compared with the name agreement proportion (Bonin et al., 2003). $H$ was computed as follows:

$H=\sum_{i=1}^{k} p_{t} \log _{2}\left(\frac{1}{p_{i}}\right)$

where $k$ refers to the different names that the subjects used for the same concept and $p$ is the ratio of the subjects who used the same name (Snodgrass \& Vanderwart, 1980). Naming failures were not considered in estimating the $H$ index.

3. Image agreement, conceptual familiarity, visual complexity, and age of acquisition were measured on a 5-point scale.

4. Naming failures were categorized into two groups: "don't know object" (DKO), where the subject failed to 
Table 2 Reliability coefficients for all measurements in middle-aged and elderly subjects

\begin{tabular}{lll}
\hline & \multicolumn{2}{l}{ Reliability Coefficient } \\
\cline { 2 - 3 } & Middle-Aged & Elderly \\
\hline Name agreement & .94 & .89 \\
Image agreement & .92 & .94 \\
Familiarity & .88 & .85 \\
Age of acquisition & .91 & .87 \\
Visual complexity & .90 & .91 \\
\hline
\end{tabular}

recognize the picture, and "don't know name" (DKN), where the subject recognized the picture but did not remember its name.

\section{Data analysis}

The pictures were considered as the units of measurement. The normality and equality of variances were examined for all of the measurements in the two age groups. The results of Kolmogorov-Smirnov and Levene's tests revealed that not all of the measurements had normal distributions in the groups. Thus, nonparametric tests were used to analyze the data. In this regard, the Mann-Whitney $U$ test was employed to examine the scales in the two groups. A comparison of the data from young subjects in the study by Bakhtiar et al. (2013) with those of the middle-aged and elderly of the present study, performed on 134 pictures that the studies had in common, was performed by Kruskal-Wallis test. Parametric statistics were used to recheck the results of the nonparametric analyses. Owing to the large number of comparisons, the level of significance was set at .01. Spearman's correlation test was employed to examine the relationships among the measurements.

\section{Results and discussion}

Description of normal data

First, the Schock, Cortese, Khanna, and Toppi (2012) method was used to determine the outliers. The mean score of each item - that is, the score given to each concept by the subjects-was calculated. Then the correlation between each subject's ratings and the overall mean was calculated. After that, the mean and standard deviation of the above-mentioned correlations were obtained. Finally, subjects whose correlations were two or more standard deviations below the overall mean of the correlation were eliminated. This procedure was done for all of the measures and for each age group separately. Accordingly, two subjects were dropped from each age group. To determine the reliability of the ratings regarding name agreement, Kuder-Richardson's Formula 20 was used, and for other items, Cronbach's alpha was employed. The obtained values were higher than .85 in all the cases (see Table 2).

The obtained data are included in a supplemental online Appendix file, in which the proportion of name agreement and $H$ index are illustrated, along with the mean scores of image agreement, conceptual familiarity, age of acquisition, and visual complexity, as well as DKN and DKO values for every concept in each age group. The online Appendix also includes the frequencies of the words. The items have been organized alphabetically. It should be noted that some Persian words have no equivalent words in English, and also an English word could sometimes refer to a few words in Persian. Table 3 illustrates a summary of the statistical data obtained for name agreement ( $H$ index and the proportion of subjects that produced each modal name), image agreement, conceptual familiarity, visual complexity, age of acquisition, DKO, and DKN.

The modal names were not determined for the words "blouse" and "goose" in the middle-aged group, and for the words "blouse," "goose," "hive," and "meat" in the elderly

Table 3 Summary statistics for all middle-aged and elderly subjects for the 272-picture set

\begin{tabular}{|c|c|c|c|c|c|c|c|c|c|c|c|c|c|}
\hline & & \multicolumn{2}{|c|}{$\begin{array}{l}\text { Name Agreement } \\
\text { (proportion) }\end{array}$} & \multicolumn{2}{|c|}{$\begin{array}{l}\text { Name } \\
\text { Agreement } \\
(H)\end{array}$} & \multicolumn{2}{|c|}{ Image Agreement } & \multicolumn{2}{|c|}{ Familiarity } & \multicolumn{2}{|c|}{ Age of Acquisition } & \multicolumn{2}{|c|}{ Visual Complexity } \\
\hline & & $\begin{array}{l}\text { Middle- } \\
\text { Aged }\end{array}$ & Elderly & $\begin{array}{l}\text { Middle- } \\
\text { Aged }\end{array}$ & Elderly & $\begin{array}{l}\text { Middle- } \\
\text { Aged }\end{array}$ & Elderly & $\begin{array}{l}\text { Middle- } \\
\text { Aged }\end{array}$ & Elderly & Middle-Aged & Elderly & Middle-Aged & Elderly \\
\hline Mean & & .90 & .80 & 0.61 & 0.80 & 4.4 & 4.5 & 3.31 & 3.24 & 1.6 & 2.11 & 1.99 & 2.22 \\
\hline Median & & .95 & .85 & 0.50 & 0.81 & 4.53 & 4.64 & 3.23 & 3.23 & 1.34 & 1.98 & 1.98 & 2.07 \\
\hline Std. D & & .14 & .18 & 0.56 & 0.65 & 0.46 & 0.46 & 1.07 & 1.15 & 0.65 & 1.01 & 0.78 & 0.80 \\
\hline Skewness & & -1.42 & -1.03 & 0.85 & 0.44 & -1.78 & -1.68 & -0.026 & -0.15 & 1.31 & 1.11 & 0.65 & 0.52 \\
\hline \multirow[t]{2}{*}{ Percentiles } & $\% 25$ & .80 & .68 & 0.09 & 0.19 & 4.22 & 4.34 & 2.34 & 2.14 & 1.00 & 1.23 & 1.34 & 1.56 \\
\hline & $\% 75$ & 1 & .97 & 0.95 & 1.29 & 4.69 & 4.82 & 4.14 & 4.13 & 1.98 & 2.56 & 2.45 & 2.98 \\
\hline
\end{tabular}


Table 4 Comparison of measurement values in the two groups via Mann-Whitney $U$ tests

\begin{tabular}{|c|c|c|c|c|c|c|c|c|}
\hline & $H$ Index & Name Agreement & Image Agreement & Familiarity & Age of Acquisition & Visual Complexity & DKN & DKO \\
\hline Mann-Whitney $U$ & $3.098 \mathrm{E} 4^{* *}$ & $2.883 \mathrm{E} 4^{* *}$ & $2.996 \mathrm{E} 4^{* *}$ & $3.593 \mathrm{E} 4^{* *}$ & $2.509 \mathrm{E} 4^{* *}$ & $3.059 \mathrm{E} 4^{* *}$ & $3.119 \mathrm{E} 4^{* *}$ & $3.481 \mathrm{E} 4^{\dagger}$ \\
\hline
\end{tabular}

group. In either group, two alternative words were used for "blouse" and "goose" that were visually similar to the target words. In the elderly group, a synonym was used for "hive," whereas a visually similar word was used for the word "meat."

As is shown in Table 3, two measures were computed for name agreement: the $H$ index and the proportion of individuals who produced modal names. When all of the subjects produced a modal name, the $H$ index was equal to 0 . However, an increased $H$ index denotes a decreased proportion of modal name production. In both the groups, the modal name frequency had a high mean score and negative skewness, whereas the $H$ index distribution was low and positively skewed. These findings suggest that the concepts had high name agreement and that many individuals correctly responded to the pictures. The $H$ index was higher for the elderly group, which indicated that their name agreement differed from that of the middleaged subjects, who used more diverse names for the pictures. Forty pictures in the middle-aged group and 42 pictures in the elderly group had an $H$ index of 0 . The alternative names used instead of the target words were synonym (e.g., "blade" instead of "knife"), superordinates (e.g., "insect" instead of "ant"), or subordinates (e.g., "villa house" instead of "house"). Some alternative names were only clipped forms of the target words, such as "pin" instead of "safety pin."

\section{Comparison of normative data between the two groups}

The obtained normative data were compared between the two age groups (Tables 4 and 5).

On the basis of Mann-Whitney $U$ tests and $t$ tests, the differences between the two groups in the proportions of mean name agreement [NA (\%)], $H$ index, image agreement, age of acquisition, visual complexity, and DKN were significant, and the differences in conceptual familiarity and DKO were nonsignificant.

Name agreement We found a significant difference between the two groups in terms of NA $(\%)$ and $H$ index. The mean NA (\%) was higher and the mean $H$ index was lower for the middle-aged group, when compared with those values for the elderly subjects, which indicated that the middle-aged subjects had higher agreement in naming concepts and used less diverse names for a given concept. Schock et al. (2012) reported similar findings on Canadian francophones.

The difficulty in finding the right word at the right time has been repeatedly reported to exist in the elderly. Recent findings have also revealed that as one grows older, the possibility of an increase in word retrieval problems rises. This might be owing to neural atrophy, which reduces cognitive activities (Shafto, Stamatakis, Tam, \& Tyler, 2010). Hodgson and Ellis (1998) assumed that naming problems in the elderly are related to overall decreased activity among pictorial, semantic, and phonological representations. Besides, in the elderly people, it is likely that the concepts eliciting various names cause the activation reaching the lexical level from the semantic level to be split among these names.

Image agreement A significant difference was also apparent in the mean scores of image agreement between the two groups: The mean score of image agreement was higher for the elderly group. The middle-aged subjects provided more comments on the pictures and noticed minute differences. Investigations showed that the evaluation procedure played a role in this difference. In the image agreement evaluation, the name of the image was revealed to the subjects, which might have caused the elderly to rate the images higher.

Conceptual familiarity No significant difference emerged in the mean scores of conceptual familiarity between the two groups. This result is inconsistent with the findings of Sirois et al. (2006), who reported that conceptual familiarity in the elderly was different from that in young and middle-aged subjects. Familiarity has a high correlation with other factors, such as frequency, which might perhaps be the reason for the

Table 5 Comparison of measurement values in the two groups via independent $t$ tests

\begin{tabular}{lllll}
\hline & \multicolumn{3}{l}{ Groups } & \multicolumn{2}{l}{$t$} & $d f$ \\
\cline { 2 - 3 } & Middle-Aged & Elderly & & \\
\hline$H$ index & $0.61(0.56)$ & $0.80(0.65)$ & $-3.709^{* *}$ & 542 \\
Name agreement & $.90(.14)$ & $.80(.18)$ & $5.076^{* *}$ & \\
Image agreement & $4.4(0.46)$ & $4.5(0.46)$ & $-2.730^{* *}$ & \\
Familiarity & $3.31(1.07)$ & $3.24(1.15)$ & $0.655^{\dagger}$ & \\
Age of acquisition & $1.6(0.65)$ & $2.11(1.01)$ & $-7.038^{* *}$ & \\
Visual complexity & $1.99(0.78)$ & $2.22(0.52)$ & $-3.321^{* *}$ & \\
DKN & $0.81(1.9)$ & $1.58(2.64)$ & $-3.924^{* *}$ & \\
DKO & $1.99(3.45)$ & $1.75(3.36)$ & $0.817^{\dagger}$ & \\
\hline
\end{tabular}

${ }^{* *} p \leq .01,{ }^{\dagger} p>.01$. Standard deviations appear in parentheses 
Table 6 Matrix of correlations among the reported measures in middle-aged subjects

\begin{tabular}{|c|c|c|c|c|c|c|c|c|}
\hline & $\begin{array}{l}\text { Name } \\
\text { Agreement } \\
(H)\end{array}$ & $\begin{array}{l}\text { Image } \\
\text { Agreement }\end{array}$ & Familiarity & $\begin{array}{l}\text { Age of } \\
\text { Acquisition }\end{array}$ & $\begin{array}{l}\text { Visual } \\
\text { Complexity }\end{array}$ & DKN & $\mathrm{DKO}$ & Frequency \\
\hline Name agreement (prop.) & $-.973^{* *}$ & $.599^{* *}$ & $.401^{* * *}$ & $-.302^{* *}$ & $-.430^{* *}$ & $-.368^{* *}$ & $-.501^{* *}$ & $.189^{* *}$ \\
\hline Name agreement $(H)$ & & $-.575^{* *}$ & $-.363^{* *}$ & $.285^{* *}$ & $.431^{* *}$ & $.288^{* *}$ & $.405^{* *}$ & $-.168^{* *}$ \\
\hline Image agreement & & & $.255^{* *}$ & $-.254^{* *}$ & $-.278^{* *}$ & $-.404^{* *}$ & $-.595^{* *}$ & $.199^{* *}$ \\
\hline Familiarity & & & & $-.482^{* *}$ & $-.318^{* *}$ & $-.342^{* *}$ & $-.284^{* *}$ & $.223^{* *}$ \\
\hline Age of acquisition & & & & & $.165^{* *}$ & $.322^{* * *}$ & $.268^{* *}$ & $-.277^{* *}$ \\
\hline Visual complexity & & & & & & $.256^{* *}$ & $.219^{* *}$ & $-.016^{* *}$ \\
\hline $\mathrm{DKN}$ & & & & & & & $.485^{* *}$ & $-.195^{* *}$ \\
\hline DKO & & & & & & & & $-.184^{* *}$ \\
\hline
\end{tabular}

${ }^{* *} p \leq .01$

lack of difference as well as for the similarity between the two groups regarding experience with these concepts.

Age of acquisition We observed a significant difference in age of acquisition between the two groups: The middle-aged subjects conjectured that they had learned the concepts at younger ages. In fact, concepts are learned over the course of life, so people may arrive at concepts at different ages. This finding is consistent with the results of Cuetos et al. (2012) on Spanish-speaking adults and Sirois et al. (2006) on Canadian francophones. The importance of age of acquisition can be determined through various tasks such as picture naming, lexical decision, and eyetracking methodology (Juhasz, 2005). Age of acquisition contributes to the prediction of retention and loss of words in cerebral disorders, and is the most reliable variable in language action (Laganaro et al., 2006). The importance of this finding lies in the fact that it demonstrates that with the passage of time and cultural and environmental changes, the age of word acquisition is changed. This difference must be taken into consideration in studies on word retrieval and treatment of naming disorders.

Visual complexity There was a significant difference in this measure between the two groups; the mean score of visual complexity was lower for the middle-aged group, when compared with that for the elderly group. This result is also inconsistent with the findings of Sirois et al. (2006), who reported no significant difference in visual complexity between their different age groups. Morrison et al. (1992) demonstrated that visual complexity affects older adults more than younger ones with respect to image naming. In addition, they also showed that visual identification of an image by an elderly person is slower, indicating that visual complexity is a factor affecting reaction times.

$D K N$ and $D K O$ The mean scores for DKN responses were significantly different between the two groups, whereas no significant difference was apparent in the mean scores for DKO responses between the two groups. Considering

Table 7 Matrix of correlations among the reported measures in elderly subjects

\begin{tabular}{|c|c|c|c|c|c|c|c|c|}
\hline & $\begin{array}{l}\text { Name } \\
\text { Agreement } \\
(H)\end{array}$ & $\begin{array}{l}\text { Image } \\
\text { Agreement }\end{array}$ & Familiarity & $\begin{array}{l}\text { Age of } \\
\text { Acquisition }\end{array}$ & $\begin{array}{l}\text { Visual } \\
\text { Complexity }\end{array}$ & $\mathrm{DKN}$ & $\mathrm{DKO}$ & Frequency \\
\hline Name agreement (prop.) & $-.950^{* *}$ & $.774^{* *}$ & $.402^{* *}$ & $-.266^{* *}$ & $-.506^{* *}$ & $-.490^{* *}$ & $-.517^{* *}$ & $.167^{* *}$ \\
\hline Name agreement $(H)$ & & $-.728^{* *}$ & $-.380^{* *}$ & $.243^{* *}$ & $.517^{* *}$ & $.370^{* *}$ & $.385^{* *}$ & $-.140^{*}$ \\
\hline Image agreement & & & $.252^{* *}$ & $-.207^{* *}$ & $-.332^{* *}$ & $-.500^{* *}$ & $-.570^{* *}$ & $.218^{* *}$ \\
\hline Familiarity & & & & $-.375^{* *}$ & $-.275^{* *}$ & $-.359^{* *}$ & $-.185^{* *}$ & $.236^{* *}$ \\
\hline Age of acquisition & & & & & $.179^{* *}$ & $.326^{* *}$ & $.122^{* *}$ & $-.334^{* *}$ \\
\hline Visual complexity & & & & & & $.309^{* *}$ & $.202^{* *}$ & .000 \\
\hline DKN & & & & & & & $.509^{* *}$ & $-.231^{* *}$ \\
\hline DKO & & & & & & & & -.113 \\
\hline
\end{tabular}

${ }^{* *} p<.01,{ }^{*} p<.05$ 
Table 8 Matrix of correlations among measures in the present study and in Sirois et al. (2006)

${ }^{* *} p<.01,{ }^{*} p<.05$

\begin{tabular}{llll}
\hline & Name Agreement (\%) & Familiarity & Visual Complexity \\
\hline Sirois \& present study (middle-aged) & $.273^{* *}$ & $.636^{* *}$ & $.385^{* *}$ \\
Sirois \& present study (elderly) & $.254^{*}$ & $.616^{*}$ & $.436^{* *}$
\end{tabular}

DKN, the retrieval of phonological features decreases with increasing age, so that even though an individual may retrieve the properties of the intended concept, he or she may be unable to utter the word. Previous studies have supported the effect of age on the phonological retrieval of a word. With increasing age, the ability of phonological retrieval decreases (Taylor \& Burke, 2002). Thus, the individual can talk about the syntactic and semantic characteristics of the word, but he or she may have access only to a part of the phonological features of the word, such as phonemes or initial syllables (Burke \& Shafto, 2004).

\section{Correlations among the measurements}

Tables 6 and 7 illustrate the correlations among all of the measurements of both the groups. As expected, we observed negative correlations between the groups with respect to NA (\%) and $H$ index, because the increase in the $H$ index indicates the extent of disagreement, whereas the increase in the values of name agreement shows the extent of agreement. Alario et al. (2004) assumed that name agreement is effective in the stages of concept selection and word selection. Therefore, words with lower name agreement proportions (i.e., higher $H$ indexes) result in more lexical candidates for naming, and the person has to choose one from among these candidates. This causes more errors and prolongation of retrieval times (Cheng, Schafer, \& Akyürek, 2010).

Age of acquisition is a strong factor that affects accuracy and speed of naming. This measurement was positively correlated to NA (\%), but negatively correlated to $H$ index in both of the groups, suggesting that there is more agreement on object names that are learned early in life. Age of acquisition seems to have an impact on the power of relation among semantic, lexical, and phonological representations, and words acquired in the early stages have stronger and more semantic relations. As a result, these words could be retrieved with greater accuracy and speed. In other words, with the increase in the age of acquisition, NA (\%) decreased in both groups. We found a negative correlation between age of acquisition and frequency, which indicated that words acquired early tended to occur more frequently in language use.

The correlation between conceptual familiarity and frequency was positive, which indicated that conceptual familiarity increased with increasing frequency. It seems obvious that more-frequent images become more familiar in daily life. Yet, Gernsbacher (1984) considered familiarity to be a more appropriate factor than the frequency of written words in investigations of lexical representations.

In speech production stages, conceptual familiarity plays a significant role in visual identification and semantic representation, and frequency is effective in all the stages of speech. Therefore, these two measurements are obviously correlated. Funnell and Davies (1996) addressed the significant role of conceptual familiarity in the prediction of naming accuracy in disorders, demonstrating that frequency control alone is not sufficient. The results of the present study showed a negative correlation between conceptual familiarity and DKN, which indicated that the more familiar a concept is, the more it is convenient to remember. Given the fact that the visual and semantic processing of more familiar concepts occurs more easily and quickly, these words are less influenced by age.

Consistent with the findings reported in previous studies (Berman, Friedman, Hamberger, \& Snodgrass, 1989; Snodgrass \& Vanderwart, 1980), the results of the present study showed a negative correlation between conceptual

Table 9 Summary statistics for young, middle-aged, and elderly subjects for 134 pictures common to the present study and Bakhtiar et al. (2013)

\begin{tabular}{|c|c|c|c|c|c|c|c|c|c|c|c|c|c|c|c|}
\hline & \multicolumn{3}{|c|}{ Name Agreement (\%) } & \multicolumn{3}{|c|}{ Name Agreement $(H)$} & \multicolumn{3}{|c|}{ Image Agreement } & \multicolumn{3}{|c|}{ Familiarity } & \multicolumn{3}{|c|}{ Visual Complexity } \\
\hline & Young & $\begin{array}{l}\text { Middle- } \\
\text { Aged }\end{array}$ & Elderly & Young & $\begin{array}{l}\text { Middle- } \\
\text { Aged }\end{array}$ & Elderly & Young & $\begin{array}{l}\text { Middle- } \\
\text { Aged }\end{array}$ & Elderly & Young & $\begin{array}{l}\text { Middle- } \\
\text { Aged }\end{array}$ & Elderly & Young & $\begin{array}{l}\text { Middle- } \\
\text { Aged }\end{array}$ & Elderly \\
\hline Mean & .93 & .91 & .85 & 0.18 & 0.41 & 0.54 & 4.1 & 4.5 & 4.64 & 4.1 & 3.67 & 3.59 & 1.21 & 1.81 & 2.01 \\
\hline Median & .99 & .95 & .91 & 0.029 & 0.22 & 0.34 & 2.2 & 4.57 & 4.73 & 4.2 & 3.78 & 3.82 & 2.1 & 1.57 & 1.98 \\
\hline Std. D & .12 & .12 & .15 & 0.33 & 0.46 & 0.54 & 0.30 & 0.34 & 0.29 & 0.51 & 1.01 & 1.08 & 0.51 & 0.73 & 0.71 \\
\hline
\end{tabular}


Table 10 Comparison of measurement values in young, middle-aged, and elderly subjects via Kruskal-Wallis tests

\begin{tabular}{|c|c|c|c|c|c|}
\hline & $H$ Index & Name Agreement & Image Agreement & Familiarity & Visual Complexity \\
\hline Chi-square & $40.92^{* *}$ & $271.79^{* *}$ & $132.58^{* *}$ & $19.89^{* *}$ & $29.56^{* *}$ \\
\hline
\end{tabular}

${ }^{* * *} p \leq .01$

familiarity and visual complexity, indicating that conceptual familiarity increased when the picture was visually simpler. This correlation might have been influenced by the effect of the degree of conceptual familiarity on speech production stages. As we mentioned earlier, conceptual familiarity is a factor that affects the speed and accuracy of visual and semantic processing. With increasing familiarity, the retrieval of a word will become quicker and more accurate, which may influence visual perception, making the image of the word appear simpler. However, this notion requires further investigation.

DKN and DKO were negatively correlated to NA (\%), but positively correlated to $H$ index. DKN and DKO were positively correlated to each other, which indicated that the pictures that were difficult to name were unclear concepts. Interestingly, the results revealed a negative correlation between visual complexity and NA (\%), so that NA (\%) for both of the groups decreased with an increase in visual complexity. Snodgrass and Vanderwart (1980) claimed that visually complex picture may affect recognition and provoke many names. Complex pictures may trigger many lemmas, and thus may exhibit low name agreement.

Image agreement was positively correlated to NA (\%), but negatively correlated to $H$ index. It has been reported that image agreement affects the visual identification system (Cheng et al., 2010). This could have caused the pictures that were in less agreement with the subject's mental image to trigger more different lemmas, leading to less name agreement. Furthermore, there were a few nonsignificant correlations $(<.3)$ among measurements for the elderly group.

\section{Correlation with other studies}

To determine the language dependency of the variables examined in the present study, the correlation between the results obtained in this study and those reported by Sirois et al. (2006) was determined. In that previous study, Canadian French norms for black-and-white images were obtained, and sociolinguistics factors, including age, were investigated. Table 8 shows the correlations between measurements of 110 common images from the present study and from the study by Sirois et al. However, owing to the different methods used, the correlation of the age of acquisition could not be determined.

The results of this cross-linguistic investigation showed a strong relationship between the measurements of conceptual familiarity and visual complexity, and a weaker relationship between the values for name agreement. It can be argued that conceptual familiarity has a strong correlation not only with language, but also with its use (Nishimoto et al., 2005). With regard to visual complexity, it can be indicated that this measurement is constant, strong, and independent of language. In the study by Raman et al. (2014), the visual complexity obtained in the Turkish language presented high correlations with Russian, Modern Greek, and Belgian French. With respect to the weaker correlation between the name agreement results, it should be noted that culture has a strong effect on this measurement (Sirois et al., 2006). Dell'Acqua, Lotto, and Job (2000) maintained that name agreement is more dependent on language than are the other variables.

Comparison of the young, middle-aged, and elderly subjects

Using the data reported by Bakhtiar et al. (2013) for young Persian speakers, a comparison of young, middle-aged, and elderly people was performed using the Kruskal-Wallis test and analysis of variance (ANOVA), except for the age-ofacquisition results. Bakhtiar et al. used the pictures from Snodgrass and Vanderwart (1980) and obtained normative data for the 18- to 29-year-old age group, in which we found 134 common items. A summary of the means for all three groups can be found in Table 9 .

Tables 10 and 11 illustrate the results from Kruskal-Wallis tests and ANOVAs, respectively, which revealed that the mean scores were significantly different among young, middleaged, and elderly subjects.

With regard to NA (\%), there was greater agreement on the names of pictures among young people, whereas the mean $H$ index was significantly lower for the young

Table 11 Comparison of measurement values in young, middle-aged, and elderly subjects via analyses of variance

\begin{tabular}{llll}
\hline & Mean Square & $F$ & $d f$ \\
\hline$H$ index & 4.74 & $22.48^{* *}$ & 2 \\
Name agreement & $379,543.93$ & $7.201 \mathrm{E} 3^{* *}$ & \\
Image agreement & 7.06 & $71.49^{* *}$ & \\
Familiarity & 13.65 & $16.71^{* *}$ & \\
Visual complexity & 4.63 & $11.38^{* *}$ \\
\hline
\end{tabular}

$$
{ }^{* *} p \leq .01
$$


Table 12 Matrix of correlations among the reported measures in young, middle-aged, and elderly subjects

\begin{tabular}{llllll}
\hline & $\begin{array}{l}\text { Name } \\
\text { Agreement } \\
\text { (prop.) }\end{array}$ & $\begin{array}{l}\text { Name } \\
\text { Agreement } \\
(H)\end{array}$ & $\begin{array}{l}\text { Image } \\
\text { Agreement }\end{array}$ & Familiarity & $\begin{array}{l}\text { Visual } \\
\text { Complexity }\end{array}$ \\
\hline Middle-aged \& young & .075 & .054 & -.046 & .036 & .15 \\
Elderly \& young & .083 & .057 & -.031 & -.011 & .18 \\
\hline
\end{tabular}

subjects, when compared with those for the other groups, indicating that the young subjects used less diverse words and synonyms. With respect to image agreement, the mean scores for the middle-aged and elderly groups were higher than that for the young subjects. This finding is difficult to justify, but it may be related to the fact that young people are typically more accurate. The mean scores of conceptual familiarity were different among the different groups, suggesting that young subjects tended to give higher scores to more-familiar pictures and that conceptual familiarity might be closely associated with age. With regard to visual complexity, the mean scores for young subjects were lower than those for the other groups, which indicated that the pictures were visually simpler for the young people.

It is noteworthy that words undergo dramatic changes over time and that new concepts enter languages. For example, many words depend on cutting-edge technology with which young people usually deal. Table 12 illustrates the correlations between the variables for the middle-aged and young groups and the old and young groups. A weak correlation could be noted between the measurements of the different age groups, and none of the correlations were significant.

The differences observed between the present study and the one by Bakhtiar et al. (2013) may not be related to age difference alone, and other factors may also be involved. Bakhtiar et al. used color images, whereas in the present study, we used black-and-white images. Furthermore, the subjects of Backtiar et al.'s study were highly educated, whereas those in the present study were illiterate or primarily literate.

\section{Conclusion}

The present study aimed to provide normative data for pictures in middle-aged and elderly Persian speakers, which may further be used in studies on cognition, language, and memory. The findings revealed that normative data were dependent on age, and hence, the data should be normalized across different age groups. The results obtained in the present study indicated that researchers should be cautious in simply applying norms derived from young subjects to elderly ones. Moreover, the present study has provided a new database for oral naming in Persian.

Author note We express our thanks to the subjects who volunteered to take part in this study, and to Mehdi Bakhtiar for his advice. We declare no conflicts of interest.

\section{References}

Alario, F. X., Ferrand, L., Laganaro, M., New, B., Frauenfelder, U. H., \& Segui, J. (2004). Predictors of picture naming speed. Behavior Research Methods, Instruments, \& Computers, 36, 140-155. doi: 10.3758/BF03195559

Bakhtiar, M., Nilipour, R., \& Weekes, B. S. (2013). Predictors of timed picture naming in Persian. Behavior Research Methods, 45, 834 841. doi:10.3758/s13428-012-0298-6

Barry, C., Morrison, C. M., \& Ellis, A. W. (1997). Naming the Snodgrass and Vanderwart pictures: Effects of age of acquisition, frequency and name agreement. Quarterly Journal of Experimental Psychology, 50A, 560-585. doi:10.1080/783663595

Berman, S., Friedman, D., Hamberger, M., \& Snodgrass, J. (1989). Developmental picture norms: Relationships between name agreement, familiarity, and visual complexity for child and adult ratings of two sets of line drawings. Behavior Research Methods, Instruments, \& Computers, 21, 371-382. doi:10.3758/bf03202800

Bonin, P., Peereman, R., Malardier, N., Méot, A., \& Chalard, M. (2003). A new set of 299 pictures for psycholinguistic studies: French norms for name agreement, image agreement, conceptual familiarity, visual complexity, image variability, age of acquisition, and naming latencies. Behavior Research Methods, Instruments, \& Computers, 35 , 158-167. doi:10.3758/BF03195507

Burke, D. M., \& Shafto, M. A. (2004). Aging and language production. Current Directions in Psychological Science, 13, 21-24. doi:10. 1111/j.0963-7214.2004.01301006.x

Cheng, X., Schafer, G., \& Akyürek, E. G. (2010). Name agreement in picture naming: An ERP study. International Journal of Psychophysiology, 76, 130-141. doi:10.1016/j.ijpsycho.2010.03. 003

Cortese, M. J., \& Khanna, M. M. (2007). Age of acquisition predicts naming and lexical-decision performance above and beyond 22 other predictor variables: An analysis of 2,342 words. Quarterly Journal of Experimental Psychology, 60, 1072-1082. doi:10.1080/ 17470210701315467

Cuetos, F., Aguado, G., Izura, C., \& Ellis, A. W. (2002). Aphasic naming in Spanish: Predictors and errors. Brain and Language, 82, 344365. doi:10.1016/S0093-934X(02)00038-X

Cuetos, F., Samartino, T., \& Ellis, A. W. (2012). Age acquisition norms from elderly Spanish people: Characteristics and the prediction of word recognition performance in Alzheimer's disease. Psicológica, 33, 59-76. 
Cycowicz, Y. M., Friedman, D., Rothstein, M., \& Snodgrass, J. G. (1997). Picture naming by young children: Norms for name agreement, familiarity, and visual complexity. Journal of Experimental Child Psychology, 65, 171-237. doi:10.1006/jecp.1996.2356

Dell, G. S., Schwartz, M. F., Martin, N., Saffran, E. M., \& Gagnon, D. A. (1997). Lexical access in aphasic and nonaphasic speakers. Psychological Review, 104, 801-838. doi: 10.1.1.113.1439

Dell'Acqua, R., Lotto, L., \& Job, R. (2000). Naming times and standardized norms for the Italian PD/DPSS set of 266 pictures: Direct comparisons with American, English, French, and Spanish published databases. Behavior Research Methods, Instruments, \& Computers, 32, 588-615. doi:10.3758/BF03200832

Dimitropoulou, M., Dunabeitia, J. A., Blitsas, P., \& Carreiras, M. (2009). A standardized set of 260 pictures for Modern Greek: Norms for name agreement, age of acquisition, and visual complexity. Behavior Research Methods, 41, 584-589. doi:10.3758/BRM.41. 2.584

Friedmann, N., Biran, M., \& Dotan, D. (2013). Lexical retrieval and its breakdown in aphasia and evelopmental language impairment. In C. Boeckx \& K. K. Grohmann (Eds.), The Cambridge handbook of biolinguistics (pp. 350-374). Cambridge: Cambridge University Press.

Funnell, E., \& Davies, P. D. M. (1996). JBR: A reassessment of concept familiarity and a category-specific disorder for living things. Neurocase, 2, 461-474. doi:10.1080/13554799608402422

Gernsbacher, M. A. (1984). Resolving 20 years of inconsistent interactions between lexical familiarity and orthography, concreteness, and polysemy. Journal of Experimental Psychology: General, 113, 256281. doi:10.1037/0096-3445.113.2.256

Hassani, H. (2005). The most frequent words of today persian, based on a one-million-word corpus: Vāže-hā-ye Porkārbord-e Fārsi-e Emruz [in Persian]. Tehran: Iran Language Institute.

Hirsh, K. W., \& Funnell, E. (1995). Those old, familiar things: Age of acquisition, familiarity and lexical access in progressive aphasia. Journal of Neurolinguistics, 9, 23-32. doi:10.1016/0911-6044(95) 00003-8

Hodgson, C., \& Ellis, A. W. (1998). Last in, first to go: Age of acquisition and naming in the elderly. Brain \& Language, 64, 146-163. doi:10. 1006/brln.1998.1960

Juhasz, B. J. (2005). Age-of-acquisition effects in word and picture identification. Psychological Bulletin, 131, 684-712. doi:10.1037/ 0033-2909.131.5.684

Laganaro, M., Di Pietro, M., \& Schnider, A. (2006). What does recovery from anomia tell us about the underlying impairment: The case of similar anomic patterns and different recovery. Neuropsychologia, 44, 534-545. doi:10.1016/j.neuropsychologia.2005.07.005

Levelt, W. J., Roelofs, A., \& Meyer, A. S. (1999). A theory of lexical access in speech production. Behavioral and Brain Sciences, 22, 138. doi: $10.1017 / \mathrm{S} 0140525 \mathrm{X} 99001776$

Morrison, C., Ellis, A., \& Quinlan, P. (1992). Age of acquisition, not word frequency, affects object naming, not object recognition. Memory \& Cognition, 20, 705-714. doi:10.3758/bf03202720

Nishimoto, T., Miyawaki, K., Ueda, T., Une, Y., \& Takahashi, M. (2005). Japanese normative set of 359 pictures. Behavior Research Methods, 37, 398-416. doi:10.3758/bf03192709
Park, D. C., \& Gutchess, A. H. (2002). Aging, cognition, and culture: A neuroscientific perspective. Neuroscience \& Biobehavioral Reviews, 26, 859-867. doi:10.1016/S0149-7634(02)00072-6

Pompeia, S., Miranda, M. C., \& Bueno, O. F. (2001). A set of 400 pictures standardised for Portuguese: Norms for name agreement, familiarity and visual complexity for children and adults. Arquivos de Neuropsiquiatria, 59B, 330-337. doi:10.1590/S0004282X2001000300004

Raman, I., Raman, E., \& Mertan, B. (2014). A standardized set of 260 pictures for Turkish: Norms of name and image agreement, age of acquisition, visual complexity, and conceptual familiarity. Behavior Research Methods, 46, 588-595. doi:10.3758/s13428-013-0376-4

Schock, J., Cortese, M., Khanna, M., \& Toppi, S. (2012). Age of acquisition estimates for 3,000 disyllabic words. Behavior Research Methods, 44, 971-977. doi:10.3758/s13428-012-0209-x

Severens, E., Van Lommel, S., Ratinckx, E., \& Hartsuiker, R. J. (2005). Timed picture naming norms for 590 pictures in Dutch. Acta Psychologica, 119, 159-187. doi:10.1016/j.actpsy.2005.01.002

Shafto, M. A., Stamatakis, E. A., Tam, P. P., \& Tyler, L. K. (2010). Word retrieval failures in old age: The relationship between structure and function. Journal of Cognitive Neuroscience, 22, 1530-1540. doi: 10.1162/jocn.2009.21321

Sirois, M., Kremin, H., \& Cohen, H. (2006). Picture-naming norms for Canadian French: Name agreement, familiarity, visual complexity, and age of acquisition. Behavior Research Methods, 38, 300-306. doi:10.3758/BF03192781

Snodgrass, J. G., \& Vanderwart, M. (1980). A standardized set of 260 pictures: Norms for name agreement, image agreement, familiarity, and visual complexity. Journal of Experimental Psychology: Human Learning and Memory, 6, 174-215. doi:10.1037/02787393.6.2.174

Tallberg, I. M. (2005). The Boston Naming Test in Swedish: Normative data. Brain and Language, 94, 19-31.

Taylor, J. K., \& Burke, D. M. (2002). Asymmetric aging effects on semantic and phonological processes: Naming in the picture-word interference task. Psychology and Aging, 17, 662-676. doi:10.1037/ 0882-7974.17.4.662

Tsaparina, D., Bonin, P., \& Méot, A. (2011). Russian norms for name agreement, image agreement for the colorized version of the Snodgrass and Vanderwart pictures and age of acquisition, conceptual familiarity, and imageability scores for modal object names. Behavior Research Methods, 43, 1085-1099. doi:10.3758/s13428011-0121-9

Weekes, B. S., Shu, H., Hao, M., Liu, Y., \& Tan, L. H. (2007). Predictors of timed picture naming in Chinese. Behavior Research Methods, 39, 335-342. doi:10.3758/BF03193165

Windfuhr, G. L. (2009). Persian. In B. Comrie (Ed.), The world's major languages (pp. 445-459). New York: Routledge.

Yoon, C., Feinberg, F., Luo, T., Hedden, T., Gutchess, A. H., Chen, H. Y., \& Park, D. C. (2004). A cross-culturally standardized set of pictures for younger and older adults: American and Chinese norms for name agreement, concept agreement, and familiarity. Behavior Research Methods, Instruments, \& Computers, 36, 639-649. doi:10.3758/ BF03206545 